durchgeführten Verkokung des bei der Tieftemperaturentgasung anfallenden Halbkokses, der mit Pech vermischt und brikeltiert wird. Das Produkt ist, wie ,a auch obne weiteres zu erwarten, ein mehr oder minder ausgegarter Koks, und die Veränderung in der Zusammensetzung der Koble ist aus fo gendem Untersuchunosbefund zu entnehmen:

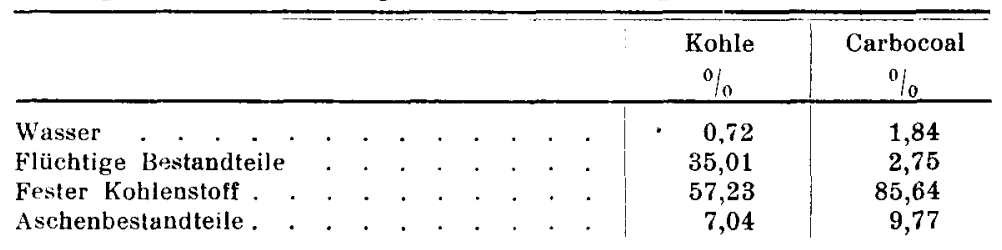

Zur Verwendung sollen in erster Linie gasreiche Kohlen gelangen, um den Prozeß als Kreisprozeß3 fübren zu könren; die Kohle wird wie im Koksproze $B$ gegrebenenfalls gewaschen und gelangt dann in Retorten, die sich in der Bauart und Wirkungsweise an die bekannte Delmonte Erverett. Retorte anlehnen und in welchen die Kohlen durch eingebaute Schneckengetriebe in Bewegung entgast werden. Die Entgasung vollzieht sich als Tieftemperaturentrasung hei einer Temperatur in den Grenzen 480-540 , eine Koksbildung wird nicht angestrebt und zufolge der Art der Entgasung auch vermieden. Was Gas selbst dient zur Keheizung der Retorten und gestatiet deren Wärmeverbrauch zu decken, wenn der Gasgehalt der verwendeten Kohle nicht zu gering ist. Nach dem völligen Erkalten wird der kleinstürkige Halbkoks (semicarbocoal) mit flüssigem Pech vermischt und die Masse dann auf Eierbriketts geformt. Das zur Verwendung gelangende Pech entstammt der Destillation des bei der Tieftemperaturentga iung anfallenden Tters. Menge und Art des Pechs müssen genau auf die Kohle eingestellt werden, um ein Zerfallen der Briketts oder ein Formverlieren derseiben bei der zweiten Verkokung zu vermeiden. Die so erzeugten PreBlinge werden dann in einer zweiten Art von Verkokungsretorten - Schiägretorten scheinen sich nach den Mitteilungen am besten bewährt zu haben - in etwa $4-5$ Stunden bei einer Temperatur von 760-1095" nochmals verkokt; sie schrumpfen, ohne aber ihre ursprïngliche Gestalt zu verlieren, so daß sehr harte, poröse, grau gefärbte Briketts erhalten werden, die eine gewisse Ähnlichkeit mit den bei uns erzeugten Koksstaubbriketts zeiven.

Das so erzeugte Carbocoal soll nicht allein im Hausbrand, sondern auch in der Industrie an Stelle von Koks verwendet werden, der Zweck des Veıfahrens ist also bis zu einem gewissen Grade der gleiche wie der des Anthracoalverfahrens, welches weiter oben besprochen wurde.

Über Menge und Beschaffenheit der bei dem neuen Verfahren ausgebrachten Produkte werden Zahlen beigebracht, die sich allerding ausschließlich auf eine in Untersuchung stehende Kohle beziehen.

Als Durchschnitt werden die Ausbeuten je $1000 \mathrm{~kg}$ trockener Kohle wie folgt angegeben:
1. Carbocoal
$725 \mathrm{~kg}$
2. Ammonsulfat $9-11 \mathrm{~kg}$
3. Andere Stickstofiverbindungen $0,056-0,113 \mathrm{~kg}$
4. Motoröl . . . . . . $6,81-8,23 \mathrm{~kg}$
5. Rohe Teeröle, hauptsächlich Ḱresole . 15,29 1
6. Wasserhelle Naphthene . . . . . . 13,24
7. Kresolöl .
20,711
8. Schweröl
17,71
9. Pech, flüssig
37,851
10. Gas mit durchschnittlich 4730 WE $254,84 \mathrm{~m}^{3}$

Pech und Gas scheiden aus der Bilanz aus, da das Pech im Betrieb weiter verwendet wird, der Wärmeverbrauch der Verkokungsöfen aber so hoch ist, daß das gesamte anfallende Gas zur Beheizung der Retorten verbraucht wird.

Das Verfahren hat zweifellos als erstes eine technisch vollbefriedigende Lösung der noch offenstehenden Frage der Formgebung für den in feiner Form anfallenden Halbkoks erbracht; wie sich seine Aussichten wirtschaftlich stellen werden, läßt sich mit Sicherheit noch nicht sagen; auf jeden Fall wird aber zu berücksichtigen sein, daß der Brennstoff doch eine ganze Reihe von Operationen durchlaufen muß und dab der Betrieb einer solchen Anlage zweifellos erheblich umständlicher und darum auch kostspieliger ist als der einer normalen Verkokungsanlage.

Für die Beurteilung der allgemeinen Verhältnisse wird aber nur die Kenntnis der Brennstoffverhältnisse des betreffenden Gebietes eine brauchbare Unterlage abgeben können. In Amerika, das über große Mengen gasreicher Kohlen verfügt, hingegen nur über eine verhältnismäßig geringe Produktion von rauchschwachverbrennenden Biennstoffen, dürfen die Verhältnisse günstio beurteilt werden: für unsere gänzlich anders gearteten Brennstoffverhältnisse dürfte das Verfahren in seiner jetzigen Form kaum Anlaß zu giinstiger Auffassung geben. Zuniichst fehlt die Möglichkeit, entsprechend große Pechmengen bei uns greifbar zu halten, da ein Großteil des Pechs bereits in die Brikettindustrie abfliebt; überdies ist auch die Art der uns zur Verarbeitung stehenden Kohlen eine ganz andere für uns, im Rahmen unserer Brennstoffwirtschaft könnte das Verlabren am ehesten noch eine Rolle zur Verwerturg des anfallenden Halbkokses aus Braunkohle spielen; on ein derartiger Prozeß unter den gegebeneı Verhältnissen aber wirtschaftlich durchgeführt werden könnte, muß nach dem derzeitigen hohen Wärmeverbrauch des Verfahrens, der dann durch zusätzlichen
Brennstoff gedeckt werden müßte, unbedingt bezweifelt werden. Die bei uns der Halbverkokung zugeführten niederwertigen Kohlen werden bei dem heutigen Stande der Tieftemperaturverkokung bestenfalls die Deckung des Wärmebedarfes der Entgasung allein sicherstellen können, auf keinen Fall aber auch die Alimentierung der bei hoher Temperatur vorzunehmenden Verkokung der Preßlinge aus Pech und Halbkokspulver. Überdies würden unsere Kohlen unter allen Umständen erbeblich höhere Aschengehalte im fertigen Carbocoal ergeben und dadurch eine nicht unbeträchtliche Wertverminderung des Fertigproduktes in Kauf nehmen müssen.

[A. 266.]

\section{Probleme der Cellulosechemie.}

\author{
Von Prof. Dr. E. Heuser ${ }^{1}$ ).
}

?(Eingeg. 16./12. 1921.)

Der Vortragende gebt nach einigen einleitenden Gedanken, wobei er- die Notwendigkeit auch rein wissenschaftlicher Forschung darlegt, bei seinen wissenschaftlichen Betrachtungen von dem technischen Prozeß der Bleiche der Cellulose aus, wobei Oxycellulose entsteht, ein Abbauprodukt der Cellulose, über deren wahre Natur wir noch sehr wenig unterrichtet sind. Vortragender erörtert die verschiedenen Auffassungen über die Natur der Oxycellulose, insbesondere auch die Frage, ob es sich hier um ein einheitliches Oxydationsprodukt oder, wie Vortragender glaubt, um ein Gemisch von geringen Mengen eines oder mehrerer Oxydationsprodukte mit unveränderter Cellulose handelt. Zwar gelingt, wie Versuche des Vortragenden mit F. Stöckigt zusammen ergeben haben, die Entfernung desjenigen Oxydationsproduktes, auf welches die Abspaltung von Kohlensäure und von Furfurol bei der Destillation mit Salzsäure zurückzuführen ist (siehe unten), und welches möglicherweise mit Glukorınsäure in Beziehung steht, erst durch-stärkeren Eingriff, nämlich durch Erhitzen mit (sehr verdünnter) Säure unter Druck, aber die Menge des so abgespaltenen Produktes ist äußerst gering; überdies erweist sich der bei dieser Behandlung verbleibende Rückstand, nämlich fast das gesamte Ausgangsprodukt, als reine Cellulose. Demnach wäre unter Oxycellulose nur eine mit geringen Mengen eines Oxydationsproduktes verunreinigte Cellulose zu versteben. Dieses Oxydationsprodukt scheint sich allerdings noch in fester Verbindung mit einem (aber ebenso geringen) Teil der Cellulose zu befinden.

Vortragender erörtert dann eingehender die von H. Pringsheim entwickelte Anschauung über die Natur der Oxycellulose, insbesondere dessen Auffassung über die Beziehungen zwischen Oxy- und Hydrocellulose, wonach die erste Stufe der Oxycellulosebildung als Hydrolyse, die zweite als eigentliche Oxydation anzusehen wäre. Die Begriffe Oxy- und Hydrocellulose gingen demnach ineinander über; erst die zweite Stufe des Vorganges erlaubt die Unterscheidung beider. Diese wird möglich durch die vor einiger Zeit von Schwalbe und Becker durchgeführte Titration der Carboxylgruppen sowie neuerdings durch die vom Vortragenden mit Stöckigt zusammen durchgefuhrte direkte Abspaltung von Kohlendioxyd aus den auf verschiedene Weise erhaltenen Oxycellulosepräparaten. Während eines dieser Präparate etwa $1,5 \% \mathrm{CO}_{2}$ abspıltete, ergab eine mittels Säuren gewonnene Hydrocellulose nur etwa $0,05 \%$; denselben geringen Wert lieferte reine Baumwollcellulose. Ebenso hält Vortragender die Abspaltung von Furfurol für ein Charakteristikum der Oxycellulose, und das Ineinandergehen von Oxy- und Hydrocellulose könnte die Ursache für die merkwürdige Erscheinung sein, daß3 die Furfurolabspaltung nicht für alle Oxycellulosepräparate charakteristisch zu sein $s^{\prime} \cdot h$ heint. Auch der Begriff Hydrocellulose wird so von neuem zum Problem, besonders auch deshalb, weil Ost darunter nicht ein Gemisch von unveränderter Cellulose und Abbauprodukten, sondern, wie er sagt ein ziemlich einheitliches Abbauprodukt der Cellulose, allerdings mit kleinerem Molekül als diese, versteht - . eine Ansicht, der sich Vortragender nicht anschließen kann.

Das Problem der Oxy- und Hydrocellulose steht in engem $\mathrm{Zu}$ sammenhang mit den Vorstellungen, die man sich von dem Aufbau der Cellulose zu machen hat. Vortragender erörtert die verschiedenen Prinzipien, nach denen die Anhäufung der Cellulosebausteine zum Cellulosemolekül denkbar ist. Er glaubt, daß wir das Prinzip der Kondensation verlassen miissen, und nimmt das der Polymerisation hierfür in Anspruch. Dafür sprechen unter anderem die Ergebnisse von Herzog und Polaniy und von Kurt $\mathrm{HeB}$, welcher zu einem Depolymerisationsprodukt der Cellulose gelangte, ohne daß ein Abbau im chemischen Sinne eingetreten wäre. Vortragender hält es für möglich, daß wir es auch in der mercerisierten Cellulose mit einem solchen Depolymerisationsprodukt $\% u$ tun haben, also mit einer lediglich physikalisch veränderten Cellulose. Auf €ine Erörterung der verschiedenen Konstitutionsformeln der Cellulose, welche die Struktur des polymerisierbaren Grundkörpers (der Anbydrocellobiose oder der Anhydroglukose) zugrunde legen, verzichtet Vortragender, da man sich hier noch zu sehr auf dem unsicheren Boden der Spekulation bewegt.

[A. 276 .

1) Da der auf S. 610 des laufenden Jahrganges der Angew. Chemie wiedergegebene Bericht einige Unrichtigkeiten enthält, bringen wir nachstehend den Eigenbericbt des Herrn Vortragenden.
Schriftleitung. 